\title{
Institute of Mathematical Sciences
}

MAGNETO-FLUID DYNAMICS DIVISION

MF -13

NYO-9492

THE EFFECT OF TEMPERATURE ON THE WIDTH OF A SMALI-AMPLITUDE, SOLITARY WAVE IN

A COLLISION-FREE PIAASMA

C. S. Garaner and G. K. Morikawa

March 15, 1961

AEC Research and Development Report 


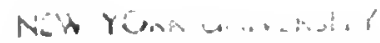

COLRANT INSTITUTE - LIERARY

$25 t$ Merre S. New York, N.Y. 10013

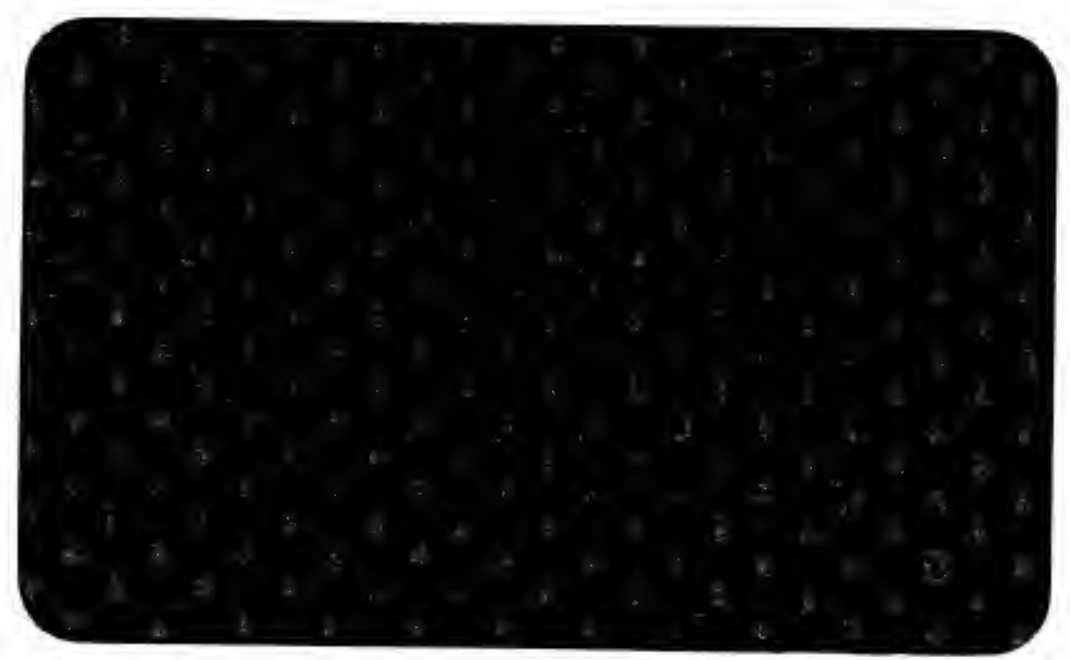

This report was prepared as an account of Government sponsored work. Neither the United States, nor the Commission, nor any person acting on behalf of the Commission:

A. Makes any warranty or representation, express or implied, with respect to the accuracy, completeness, or usefulness of the information contained in this report, or that the use of any information, apparatus, method, or process disclosed in this report may not infringe privately owned rights; or

B. Assumes any liabilities with respect to the use of, or for damages resulting from the use of any information, apparatus, method, or process disclosed in this report.

As used in the above, "person acting on behalf of the Commission" includes any employee or contractor of the Commission, or employee of such contractor, to the extent that such employee or contractor of the Commission, or employee of such contructor prepares, disseminates, or provides access to, any information pursuant to his employment or contract with the Commission, or his employment with such contractor. 


\section{Magneto-Fluid Dynamics Division Institute of Mathematical Sciences New York University}

Physics and Mathematics

MF -13 NYO-9492

THE EFFECT OF TEMPERATURE ON THE WIDTH OF A SMALL-AMPLITUDE, SOLITARY WAVE IN A COLLISION-FREE PILASMA

C. S. Garáner and G. K. Morikawa March 15, 1961

AEC Research and Development Report

Contract No. AT (30-1)-1480 



\section{Abstract}

Adlam and Allen [1] and Davis, Lthst, and Schlther [4] have studied nonlinear plane-waves, propajating normal to the magnetic field, in a cold plasma. One solution of particular interest is a solitary wave, or single pulse. We present a method for solving the analogous problem for a plasma with finite temperature, in the limiting case where the amplitude of the wave is small -- and where, consequently, the width of the wave is very large. Our result is that in a warm plasma the solitary wave of small amplitude has the same form as in a cold plasma, but has a width which is larger than its width in a cold plasma by a factor which is approximately

$$
\sqrt{1+\frac{1}{16}\left(\frac{m+}{m}\right) \beta} .
$$

Here $\beta$ is the ratio of material pressure to magnetic pressure in the undisturbed plasma; and $m_{+}, m_{-}$are the respective masses of a positive ion and of an electron. Evidently this factor is appreciable, unless $\beta$ is very small. 

Table of Contents

\section{Page}

Abstract

2

Section

1. Introduction

4

2. Equations of Motion. Dimensionless Variables and Scaling Transformations

6

3. Series Solution of the Collision-free Boltzmann Equations.

4. Computation of the Moments

19

5. Differential Equation for B 22

6. Width of the Solitary wave 25

References

26 

The Effect of Temperature on the Width of a Small-Amplitude, Solitary wave in a Collision-Free Plasma

1. Introduction.

Adlam and Allen [1] and Davis, Llist, and Schliter [4] have studied the nonlinear steady plane waves which can propagate normal to the magnetic field in a collision-free plasma. These authors assumed a cold plasma -- in which the temperature of the positive-ion gas is zero, and the temperature of the electron gas is zero. Thus at any point, in a cold plasma, all ions have the same velocity, and all electrons have the same velocity. A one-dimensional steady flow of a cold plasma is therefore governed by ordinary differential equation. These equations were solved by Adlam and Allen, and, in fuller detail, by Davis, Litst, and Schluter. In general the solution represents a periodic wave train (which is not sinusoldal, in this nonlinear theory). A particular solution, of special interest, represents a single pulse, or solitary wave. Gardner, et al. [5], Rose [14], Banos and Vernon [2] and Vernon [15] have obtalned similar solutions from various two-fluid theorles (based on suggestions due to Grad, [7] and [8]) which include pressure and temperature. Our object is to investigate the effect of finite plasma temperature on the solitary wave solution. If no approximations are made, (other than that the plasma is collision-free), the equations of motion are too difficult to be solved for a warm plasma (with temperature). We are able, however, to find a solution in the limiting case of a wave of small amplitude. Our 
approximation here does not lead to a linear theory; a linear theory could never yield a solitary wave as solution. "The reason is that the width of the solitary wave tends to infinity as the amplitude tends to zero; so that the independent variable must be scaled accordingly. In fact, we assume the same scaling laws for the warm plasma as obtains for the solitary wave in the cold plasma. That is, we assume that the width of the wave varies inversely as the square root of its amplitude. This was shown for the cold plasma by Gardner and Morikawa [6]. Thus a length over which the magnetic and electric fields vary appreciably is very large in this limit. Consequently, one can obtain a formal solution of the collision-free Boltzmann equation by a successive-approximation calculation. This calculation yields expressions for the distribution functions of the ions and electrons in terms of the electric and magnetic field components and their derivatives. Inserting these expressions into Maxwell's equations, and retaining only the terms of lowest significant order, we obtain a differential equation for the asymptotic shape or the wave. This equation has the same form as the analogous equation for the cold plasma; however the coefficients depend on the plasma temperature. The final conclusion which can be drawn is that the effect of temperature is to multiply the width of the

\footnotetext{
We point out that the inherent non-linearity (even for small amplitude) of the solitary wave solution in the study of gravity waves in water of finite depth has been known for many years (cf. Kortewer and de Vries, [10], and Keller, [9]). Other physical problems which exhibit this peculiarity are described more recently by Morikana [12], [13].
} 
Adlam-Allen solitary wave by a factor which is approximately

$$
\sqrt{1+\frac{1}{16}\left(\frac{m_{+}}{m_{-}}\right) \beta},
$$

(assuming for simplicity that the ion temperature and electron temperature are equal). Here $\beta$ is the ratio of material pressure to magnetic pressure in the undisturbed plasma; and $m_{+}$is

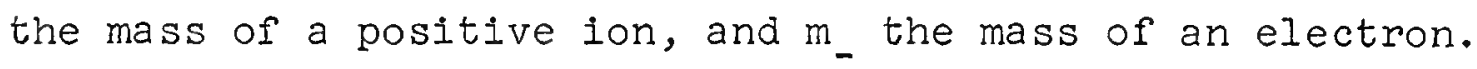
Evidently this factor is appreciable unless $\beta$ is very small. Another way of stating the result is that the cold-plasma treatment of the solitary wave is correct only if the electron sound speed is small compared to the Alfven speed in the undisturbed plasma. This result may be pertinent to the study of weak, collision-free plasma shocks (cf. Morawetz, [11]).

2. Equations of Motion. Dimensionless Variables and Scaling Transformations

Our problem is to study a plane wave, propagating with speed $U_{0}$ in the negative $\bar{x}$ direction, in a plasma, initially in equilibrium, and permeated by a constant uniform field of strength $B_{O}$, in the $\bar{z}$ direction. We shall formulate this problem in a coordinate system which moves with the wave. We assume that in this coordinate system we have a steady-state flow of the plasma. Due to the coordinate transformation there is now a constant 
electric field, in the $\bar{y}$ direction, of magnitude $E_{y}=B_{0} U_{0}$. We use m.k.s. electrical units. Here, of course, $B_{0}$ means the undisturbed magnetic field as it appears in the moving coordinate system. The plasma consists of positive ions and electrons continuously distributed. We assume that the motion of an ion or electron is determined only by the force exerted on it by the macroscopic electric and magnetic flelds. The magnetic field is entirely in the $\bar{z}$ direction, and has strength $\bar{B}(\bar{x})$ (depending only on $\bar{x}$, and independent of time, in the frame of reference fixed to the wave). The electric field has the component $\mathrm{E}_{\mathrm{y}}=\mathrm{B}_{\mathrm{O}} \mathrm{U}_{\mathrm{O}}$ and also the component $\mathrm{E}_{\mathrm{x}}(\overline{\mathrm{x}})$, due to charge separation. The plasma proper is described by the distribution functions of the ions and electrons, which are time-independent, and depend on only the one space coordinate $\bar{x}$.

Let $\bar{u}, \bar{v}$ denote the $\bar{x}$ and $\bar{y}$ components of the velocity of a particle. Let $\overline{\mathrm{f}}_{+}(\overline{\mathrm{x}}, \overline{\mathrm{u}}, \bar{v})$ and $\overline{\mathrm{f}}_{-}(\overline{\mathrm{x}}, \overline{\mathrm{u}}, \bar{v})$ denote the density of the ions and the density of the electrons in the $\bar{x} \bar{u} \bar{v}$ phase space. Then the distribution functions $\bar{f}_{+}, \bar{f}_{-}$satisfy the following collisionless Boltzmann equations:

$$
\bar{u} \frac{\partial \bar{f}_{+}}{\partial \bar{x}}+\frac{e}{m_{+}}\left(E_{x}+\bar{B} \bar{v}\right) \frac{\partial \bar{f}_{+}}{\partial \bar{u}}+\frac{e}{m_{+}}\left(E_{y}-\bar{B} \bar{u}\right) \frac{\partial \bar{f}_{+}}{\partial \bar{v}}=0
$$

$$
\bar{u} \frac{\partial \bar{f}}{\partial \bar{x}}-\frac{e}{m_{-}}\left(E_{x}+\bar{B} \bar{v}\right) \frac{\partial \bar{f}}{\partial \bar{u}}-\frac{e}{m_{-}}\left(E_{y}-\bar{B} \bar{u}\right) \frac{\partial \bar{f}}{\partial \bar{v}}=0
$$

Here $e$ is the electronic charge, and $m_{+}$is the mass of an ion, 
and $m$ - the mass of an electron. The electric and magnetic fields are governed by the Maxwell equations,

$$
\frac{d E_{X}}{d \bar{x}}=\mu c^{2} e^{2}\left[\iint \bar{f}_{+} d \bar{u} d \bar{v}-\iint \bar{f}_{-} d \bar{u} d \bar{v}\right]
$$

$$
\frac{d \bar{B}}{d \bar{x}}=-\mu e\left[\iint \bar{v} \bar{f}_{+} d \bar{u} d \bar{v}-\iint \bar{v} \bar{f}_{-} d \bar{u} d \bar{v}\right]
$$

where $\mu$ is the permeability of space and $c$ the speed of 11 ght. (We use M.K.S. units.)

We are interested in solutions which tend to a constant state as $\bar{x}$ tends to $-\infty$. That 1 , we suppose the following conditions :

$$
\text { For } \bar{x} \rightarrow-\infty \text {, }
$$

$$
\begin{array}{ll}
\text { (5) } & \bar{f}_{+}(\bar{x}, \bar{u}, \bar{v}) \rightarrow \bar{\psi}_{+}\left(\left(\bar{u}-U_{0}\right)^{2}+\bar{v}^{2}\right) \\
(6) & \bar{f}_{-}(\bar{x}, \bar{u}, \bar{v}) \rightarrow \bar{\psi}_{-}\left(\left(\bar{u}-U_{0}\right)^{2}+\bar{v}^{2}\right) \\
\text { (7) } & \bar{B}(\bar{x}) \rightarrow B_{0}(\text { a constant }) \\
\text { (8) } & E_{X}(\bar{x}) \rightarrow 0
\end{array}
$$

where

$$
E_{y}=B_{O} U_{O},
$$

and where the number densities of ions and electrons are equal:

$$
\iint \bar{\psi}_{+} d \bar{u} d \bar{v}=n_{0}=\iint \bar{\psi}_{-} d \bar{u} d \bar{v}
$$


Here $\bar{\psi}_{+}$and $\bar{\psi}_{-}$are the undisturbed distribution functions for ions and for electrons. The most interesting case, of course, is the case wherein $\bar{\psi}_{+}, \bar{\psi}_{-}$are Maxwellian with equal temperature (not a necessary restriction).

It is easily verified that one solution is given by

$$
\overline{\mathrm{f}}_{+} \equiv \bar{\psi}_{+}, \quad \overline{\mathrm{f}}_{-} \equiv \bar{\Psi}_{-}, \quad \overline{\mathrm{B}}(\overline{\mathrm{x}}) \equiv \mathrm{B}_{0}, \quad \mathrm{E}_{\mathrm{x}} \equiv 0 .
$$

This is the trivial solution -- corresponding to no disturbance of the plasma. It is not really clear than any other steady state solution does in fact exist. However, we shall assume that such a non-trivial solution exists, and furthermore that it depends asymptotically on a parameter $\epsilon$ in a certain way. We then proceed to find the solution for small $\epsilon$, correct to lowest order in $\epsilon$.

To define $\epsilon$, we first must define a few other parameters of importance. Let $p_{+}, p_{-}$denote the initial pressures of the ion gas and of the electron gas; then

$$
p_{+}=\frac{1}{2} m_{+} \iint\left[\left(\bar{u}-U_{0}\right)^{2}+\bar{v}^{2}\right] \bar{\psi}+d \bar{u} d \bar{v}
$$

$$
p_{-}=\frac{1}{2} m_{-} \iint\left[\left(\bar{u}-U_{0}\right)^{2}+\bar{v}^{2}\right] \bar{\psi}_{-} d \bar{u} d \bar{v} .
$$

Let $a$ be the sound speed, and A the Alfven speed, of the undisturbed plasma; then

$$
a^{2}=\frac{2\left[p_{+}+p_{-}\right]}{n_{0}\left[m_{+}+m_{-}\right]}
$$




$$
A^{2}=\frac{B_{0}^{2}}{\mu n_{0}\left[m_{+}+m_{-}\right]} .
$$

Let the number $M$ be defined by

$$
M^{2}=\frac{U_{0}^{2}}{A^{2}+a^{2}} .
$$

Then $M$ is akin to Mach number, being the ratio of the flow speed $U_{0}$ to the speed $\left(A^{2}+a^{2}\right)^{1 / 2}$, which is the propagation speed of a small-amplitude hydromagnetic wave normal to the magnetic field. We define $\epsilon$ by setting

$$
M^{2}=1+\epsilon^{2} \text {. }
$$

We define dimensionless variables (without bars) as follows:

$$
\bar{x}=\frac{1}{\epsilon} \frac{m_{+} U_{0}}{e B_{0}} x
$$

$$
\vec{u}=U_{0}(I+u) ; \quad \bar{v}=U_{0} v
$$

$$
\bar{B}=B_{0}\left(I+\epsilon^{2} B\right)
$$

$$
E_{X}=\epsilon^{3} B_{0} U_{0} E
$$

$$
\overline{\mathrm{f}}_{+}=\frac{\mathrm{n}_{0}}{\mathrm{U}_{0}^{2}} \mathrm{f}_{+} ; \overline{\mathrm{f}}_{-}=\frac{\mathrm{n}_{0}}{\mathrm{U}_{0}^{2}} \mathrm{f}_{-}
$$

$$
\bar{\psi}_{+}=\frac{n_{0}}{\mathrm{U}_{0}^{2}} \psi_{+}\left(u^{2}+v^{2}\right) ; \quad \bar{\psi}_{-}=\frac{n_{0}}{\mathrm{U}_{0}^{2}} \psi_{-}\left(u^{2}+v^{2}\right)
$$

The reasen for introducing the powers of $\epsilon$ into (16), (18), 
(19) is that we assume that the length scale of the disturbance is of order $1 / \epsilon$, and that the disturbance in $B$ is of order $\epsilon^{2}$, and the disturbance $\mathrm{E}_{\mathrm{X}}$ is of order $\epsilon^{3}$, as $\epsilon$ tends to zero. These scaling rules, which, as we shall see, are self-consistent, were motivated by their validity for the solitary wave in a cold plasma. The explicit solution is available for the solitary wave in a cold plasma. And, in agreement with the above, it is found that the non-trivial solution exists only if $M>1$; and for $M^{2}=1+\epsilon^{2}$, and $E_{X}$ is of order $\epsilon^{3}$, (as shown by Gardner and Morikawa).

In terms of the new variables given by (16)-(20), the Boltzmann equations take on the following forms:

$$
\frac{\partial f_{t}}{\partial \theta}+\epsilon^{2} B \frac{\partial f_{t}}{\partial \theta}=\epsilon(I+u) \frac{\partial f_{t}}{\partial x}-\epsilon^{2} B \frac{\partial f_{t}}{\partial v}+\epsilon^{3} E \frac{\partial f_{t}}{\partial u}
$$

$$
\frac{\partial f}{\partial \theta}+\epsilon^{2} B \frac{\partial f}{\partial \theta}=-\epsilon \alpha(I+u) \frac{\partial f}{\partial x}-\epsilon^{2} B \frac{\partial f}{\partial v}+\epsilon^{3} E \frac{\partial f}{\partial u} .
$$

Here $\theta$ is a polar coordinate in the $u, v$ plane; we define $w, \theta$ by

$$
\begin{aligned}
& w=u^{2}+v^{2} \\
& u=w^{1 / 2} \cos \theta, \quad v=w^{1 / 2} \sin \theta
\end{aligned}
$$

so that

$$
\text { (26) } \frac{\partial}{\partial \theta}=u \frac{\partial}{\partial v}-v \frac{\partial}{\partial u} \text {. }
$$

The coefficient $\alpha$ in (23) is the ratio of the masses of the 
two tupes of particle:

(27) $\quad \alpha=\frac{m_{-}}{m_{+}}$.

The Maxwell equations now take on the following forms:

(28) $\quad \epsilon^{4}(1+\alpha) \frac{A^{2}}{c^{2}} \frac{d E}{d x}=\left[\iint f_{+} d u d v-\iint f_{-} d u d v\right]$

(29) $\epsilon^{3}\left[-(1+\alpha)+2\left(1+\epsilon^{2}\right)\left(P_{+}+\alpha P_{-}\right)\right] \frac{d B}{d x}=\left(1+\epsilon^{2}\right)\left[\iint v f_{+} d u d v\right.$

$\left.-\iint v f_{-} d u d v\right]$

Here $\mathrm{P}_{+}$and $\mathrm{P}_{-}$ar: uimeneionless pressures defined by

$(30) \quad P_{+}=\frac{p_{+}}{n_{0} m_{+} U_{0}^{2}}, \quad P_{-}=\frac{p_{-}}{n_{0} m_{-} U_{0}^{2}}$.

Finally, we have the following conditions as $x$ tends to $-\infty$ :

$$
\text { For } x \rightarrow-\infty
$$

(31)

$$
f_{+}(x, u, v) \rightarrow \psi_{+}\left(u^{2}+v^{2}\right)
$$

$$
f_{-}(x, u, v) \rightarrow \psi_{-}\left(u^{2}+v^{2}\right)
$$

(33)

$$
\mathrm{B}(\mathrm{x}) \rightarrow 0
$$

(34)

$$
E(x) \rightarrow 0
$$

and

(35) $\iint \psi_{+} d u d v=1, \quad \iint \psi_{-} d u d v=1$ 
(36)

$$
\begin{aligned}
& \frac{1}{2} \iint\left(u^{2}+v^{2}\right) \psi_{+} d u d v=P_{+}, \\
& \frac{1}{2} \iint\left(u^{2}+v^{2}\right) \psi_{-} d u d v=P_{-} .
\end{aligned}
$$

3. Series Solution of the Collision-free Boltzmann Equations. Our procedure for solving the system (22), (23), (28), (29), with initial conditions (31)-(36) is to put

$$
\begin{aligned}
& f_{+}=f_{+}^{(0)}+\epsilon f_{+}^{(1)}+\epsilon f_{+}^{2}(2)+\ldots \\
& f_{-}=f_{-}^{(0)}+\epsilon f_{-}^{(1)}+\epsilon^{2} f_{-}^{(2)}+\ldots
\end{aligned}
$$

in the Boltzmann equations (28), (29), and equate the coefficient of each power of $\epsilon$ on the left with the coefficient of the same power of $\epsilon$ on the right in the usual way. We thereby obtain a sequence of equations, which we can solve explicitly, and we obtain $f^{(0)}, f^{(I)}$, etc. In terms of $B(x), E(x)$ and their derivatives. A different, but related scheme, is used by chew, Goldberger and Low [3], the difference being in the scale transformations. We then insert our solution of the Boltzmann equation into the Maxwell equations (28), (29) and obtain differential equations -- to any order -- $E(x), B(x)$. Retaining only the terms of lowest significant order, we finally 
arrive at an equation of third order for $B(x)$. This equation can be solved by quadratures. The solution ylelds the asymptotic shape of the solitary wave in a warm plasma.

To carry out the iteration process for solving the Boltzmann equations, we can work with the ion equation (22) only, since the results for the electron equation can be obtained from the result for the ion equation by replacing

$$
\frac{\partial}{\partial x} \quad b y \quad-a \frac{\partial}{\partial x}
$$

Thus we may consider the following:

(37)

$$
\frac{\partial f}{\partial \theta}+\epsilon^{2} B(x) \frac{\partial f}{\partial \theta}=\epsilon(I+u) \frac{\partial f}{\partial x}-\epsilon^{2} B(x) \frac{\partial f}{\partial v}+\epsilon^{3} E(x) \frac{\partial f}{\partial u}
$$

where

$$
u=v^{1 / 2} \cos \theta, \quad v=w^{1 / 2} \sin \theta
$$

Wtin the conditions for $x \rightarrow-\infty$,

(38) $\quad f(x, u, v) \rightarrow \psi(w)$

(39) $\quad B(x) \rightarrow 0$

(40) $\quad E(x) \rightarrow 0$.

We solve (37) by setting

(il) $\quad r=f_{0}+\epsilon f_{1}+\epsilon^{2} f_{2}+\epsilon^{3} f_{3}+\epsilon^{4} f_{4}+\ldots$ 
and solving the sequence of equations, corresponding to the successive powers of $\epsilon$, which we get on inserting (4I) into (37). We use as initial conditions, to determine the constants of integration, the conditions

$$
\text { for } x \rightarrow-\infty \text {, }
$$

(42)

$$
f_{0} \rightarrow \psi
$$

$\mathrm{f}_{1} \rightarrow 0$

$\mathrm{f}_{2} \rightarrow 0$, etc.

(43)

$$
B(x) \rightarrow 0,
$$

$\mathrm{B}^{\prime}(\mathrm{x}) \rightarrow 0$,

$B^{\prime \prime}(x) \rightarrow 0$, etc.

(44)

$$
E(x) \rightarrow 0,
$$

$E^{\prime}(x) \rightarrow 0$,

$E^{\prime \prime}(x) \rightarrow 0$, etc.

We shall have to compute $f_{0}, f_{1}, f_{2}, f_{3}$, and $f_{4}$ in order to obtain the lowest order approximation of the field variables $B$, etc.

In the lowest order, we have

$$
\frac{\partial f_{0}}{\partial \theta}=0
$$

whence

$$
f_{0}=\phi_{0}(x, w)
$$

where $\phi_{0}$ is yet to be determined. The function $\phi_{0}$ will be determined by a compatibility condition, for the solvability of the next-order equation for $f_{I}$. In the next order, we have (45) $\frac{\partial f_{I}}{\partial \theta}=(1+u) \frac{\partial f_{0}}{\partial x}=(1+u) \frac{\partial}{\partial x} \not_{0}(x, w)$. 
We integrate both sides with respect to $\theta$, holding $x, w$ fixed, from $\theta=0$ to $\theta=2 \pi$. We get

$$
0=\frac{\partial \not_{0}}{\partial x}
$$

Using the initial condition (42), we get

$$
\phi_{0}=\psi(w) ; \text { so that } f_{0}=\psi(w) \text {. }
$$

Now, from (45), (46) we have

$$
\frac{\partial f_{1}}{\partial \theta}=0
$$

whence

$$
f_{1}=\not \not_{1}(x, w) .
$$

Again, $\not_{1}$ will be determined from the next equation (coefficient of $\left.\epsilon^{2}\right)$.

This next equation is

$$
\text { (50) } \quad \frac{\partial f_{2}}{\partial \theta}+B \frac{\partial f_{0}}{\partial \theta}=(1+u) \frac{\partial f_{1}}{\partial x}-B \frac{\partial f_{0}}{\partial v} \text {. }
$$

Using (47), (49), this becomes

$$
\frac{\partial f_{2}}{\partial \theta}=(1+u) \frac{\partial}{\partial x} \not_{1}(x, w)+B \psi^{\prime}(w)[-2 v] .
$$

Integrating with respect to $\theta$, we get

$$
0=\frac{\partial}{\partial x} \phi_{1}(x, w) .
$$

Thus, by (42), we obtain 
(53)

$$
\phi_{1}=0 ; \text { so that } f_{1}=0 \text {. }
$$

Now (51) becomes

$$
\frac{\partial f_{2}}{\partial \theta}=B \Psi^{\prime}(w)[-2 v]
$$

whence

$$
f_{2}=B \psi^{\prime}(w)[2 u]+\not \phi_{2}(x, w) .
$$

Again, to find $\phi_{2}$ we go to the next equation (coefficient of $\epsilon^{3}$ ). The next equation is

$$
\frac{\partial f_{3}}{\partial \theta}+B \frac{\partial f_{1}}{\partial \theta}=(1+u) \frac{\partial f_{2}}{\partial x}-B \frac{\partial f_{1}}{\partial v}+E \frac{\partial f_{0}}{\partial u}
$$

or, by $(47),(53),(54)$,

$$
\frac{\partial f_{3}}{\partial \theta}=(1+u) \frac{\partial \phi_{2}}{\partial x}+B^{\prime} \psi^{\prime}(w)\left[2 u+2 u^{2}\right]+E \psi^{\prime}(w)[2 u] .
$$

Integrating (56) with respect to $\theta$, we obtain

$$
0=\frac{\partial \phi_{2}}{\partial x}+B^{\prime} w^{\prime}(w)
$$

whence

$$
\not D_{2}=-B w \psi^{\prime}(w)
$$

and, by (54),

$$
f_{2}=B \psi^{\prime}(w)[-w+2 u] .
$$

Multiplying (57) by $(1+u)$ and subtracting the result from (56), 
we get

(59)

$$
\frac{\partial f_{3}}{\partial \theta}=B^{\prime} \psi^{\prime}(w)\left[-w+(2-w) u+2 u^{2}\right]+E \psi^{\prime}(w)[2 u]
$$

We can find an integral of this equation: We make use of the facts

$$
\frac{\partial w}{\partial \theta}=0, \quad \frac{\partial}{\partial \theta}=u \frac{\partial}{\partial v}-v \frac{\partial}{\partial u} .
$$

We find

$$
f_{3}=B^{\prime} \psi^{\prime}(w)[(2-w) v+u v]+E \psi^{\prime}(w)[2 v]+\not \phi_{3}(x, w) .
$$

Again, to find $\not_{3}$ we must use the next equation (coefficient of $\left.\epsilon^{4}\right)$.

By now the nature of the algorithm should be clear. From this point on the calculations rapidly become lengthier. Therefore we shall not write out all the steps, but at this point merely give the results. We obtain the following:

$$
f_{0}=\psi
$$

$$
f_{1}=0
$$

$$
f_{2}=B \psi^{\prime}[-w+2 u]
$$

$$
f_{3}=B^{\prime} \psi^{\prime}[(2-w) v+u v]+E \psi^{\prime}[2 v]
$$

$$
\begin{aligned}
f_{4} & =B^{\prime \prime} \psi^{\prime}\left[\left(\frac{7}{4} w-\frac{5}{8} w^{2}\right)+(-2+w) u+\left(-\frac{3}{2}+\frac{1}{2} w\right) u^{2}-\frac{1}{3} u^{3}\right] \\
& +E^{\prime} \psi^{\prime}\left[\frac{3}{2} w-2 u-u^{2}\right]+B^{2} \psi^{\prime}[(1+w)-4 u] \\
& +B^{2} \psi^{\prime \prime}\left[\frac{1}{2} w^{2}-2 w u+2 u^{2}\right] .
\end{aligned}
$$


4. Computation of the Moments

We now compute the moments of $f_{+}, f_{-}$which occur in the Maxwell equations. We have

$$
\iint \psi(w) d u d v=\pi \int_{0}^{\infty} \psi(w) d w=1
$$

and we define $P, \sigma$ by

$$
2 P=\iint w \psi(w) d u d v=\pi \int_{0}^{\infty} w \psi(w) d w
$$

$$
4 \sigma \mathrm{P}^{2}=\iint w^{2} \psi(w) d u d v=\pi \int_{0}^{\infty} w^{2} \psi(w) d w .
$$

(Note that (68) is a fourth moment.) By applying Schwarz's inequality $\left(\left|\int F \cdot G\right|^{2} \leq\left|\int F^{2}\right| \cdot\left|\int G^{2}\right|\right)$ it can be shown that if $\psi$ is positive, then

$$
\sigma \geq 1
$$

and that if $\psi(w)$ is positive and a monotone decreasing function of $w$, that

$$
\sigma \geq 4 / 3
$$

If $\psi$ is a Maxwellian distribution, then

$$
\sigma=2
$$

By integrating (61) to (65) we obtain the following:

$$
\text { (69) } \iint f_{0} d u d v=1
$$


(70) $\iint f_{1} d u d v=0$
$(71) \quad \iint f_{2} d u d v=B$
$(72) \quad \iint f_{3} d u d v=0$
$(73) \quad \iint f_{4} d u d v=B^{\prime \prime}\left[-1+\frac{3}{2} P\right]-E^{\prime}+2 B^{2}$.

Also, we obtain the following:

(74) $\iint v f_{0} d u d v=0$

(75) $\iint v f_{1} d u d v=0$

(76) $\iint v f_{2} d u d v=0$

(77) $\iint v f_{3} d u d v=B^{\prime}[-1+2 P]$

(78) $\quad \iint v r_{4} d u d v=0$.

We shall also need the moment of $f_{5}$. This can be obtained, without actually computing $f_{5}$, as follows: we multiply the Boltzmann equation (37) by $u$ and integrate, noting that

$$
\begin{aligned}
& \iint u \frac{\partial f}{\partial \theta} d u d v=\iint u\left[u \frac{\partial f}{\partial v}-v \frac{\partial f}{\partial u}\right] d u d v \\
= & \iint\left[\frac{\partial}{\partial v}\left(u^{2} f\right)-\frac{\partial}{\partial u}(u v f)\right] d u d v+\iint v f d u d v \\
= & \iint v f d u d v .
\end{aligned}
$$

We obtain, then, 
$\left(1+\epsilon^{2} B\right) \iint v f d u d v=\epsilon \frac{\partial}{\partial x} \iint\left(u+u^{2}\right) f d u d v-\epsilon^{3} E \iint f d u d v$

so that

$$
\iint v f_{5} d u d v+B \iint v f_{3} d u d v=\frac{\partial}{\partial x} \iint\left(u+u^{2}\right) f_{4} d u d v-E \iint f_{2} d u d v
$$

In this way we obtain

$$
\iint v f_{5} d u d v=B^{\prime \prime} \cdot\left[1-\frac{11}{4} P+\frac{3}{2} \sigma P^{2}\right]+E^{\prime \prime}\left[1-\frac{3}{2} P\right]+3 B B^{\prime} .
$$

From these results we obtain the following:

(80)

$$
\iint f_{+} d u d v=1+\epsilon^{2} B+\epsilon^{4}\left\{B^{\prime \prime}\left[-1+\frac{3}{2} P_{+}\right]-E^{\prime}+2 B^{2}\right\}+o\left(\epsilon^{6}\right)
$$

$$
\iint f_{-} d u d v=1+\epsilon^{2} B+\epsilon^{4}\left\{\alpha^{2} B^{\prime \prime}\left[-1+\frac{3}{2} P_{-}\right]+\alpha E^{\prime}+2 B^{2}\right\}+O\left(\epsilon^{6}\right)
$$

$$
\begin{aligned}
& \iint v f_{+} d u d v=\epsilon^{3}\left\{B^{\prime}\left[-I+2 P_{+}\right]-E\right\} \\
& +\epsilon^{5}\left\{B^{\prime \prime \prime}\left[1-\frac{11}{4} P_{+}+\frac{3}{2} \sigma_{+} P_{+}^{2}\right]+E^{\prime \prime}\left[1-\frac{3}{2} P_{+}\right]+3 B B^{\prime}\right\}+O\left(\epsilon^{7}\right)
\end{aligned}
$$

$$
\begin{aligned}
& \iint v f_{-} d u d v=\epsilon^{3}\left\{B^{\prime}\left[\alpha-2 \alpha P_{-}\right]-E\right\} \\
& +\epsilon^{5}\left\{B^{\prime \prime} \cdot\left[-\alpha^{3}+\frac{11}{4} \alpha^{3} P_{-}-\frac{3}{2} \alpha^{3} \sigma_{-} P_{-}^{2}\right]+E^{\prime \prime}\left[\alpha^{2}-\frac{3}{2} \alpha^{2} P_{-}\right]\right. \\
& \left.-3 \alpha B B^{\prime}\right\}+o\left(\epsilon^{7}\right) .
\end{aligned}
$$

These moments are sufficient for our purpose in the following sections. However, an appreciable amount of additional calculations will be necessary in order to obtain an explicit expression for the 
anisotropic pressure tensor corresponding to our distribution function. Then a more direct comparison of our results with other two-fluid theories will be possible.

5. Differential Equation for B

If we insert the expressions (80), (81), (82), (83) into the Maxwell equations (28), (29) we obtain the following:

(84)

$$
\begin{aligned}
& (1+\alpha) \frac{A^{2}}{c^{2}} E^{\prime}=B^{\prime \prime}\left[-\left(1-\alpha^{2}\right)+\frac{3}{2}\left(P_{+}-\alpha^{2} P_{-}\right]-(1+\alpha) E^{\prime}+O\left(\epsilon^{2}\right)\right. \\
& {\left[-(1+\alpha)+\left(1+\epsilon^{2}\right)\left(2 P_{+}+2 \alpha P_{-}\right)\right] B^{\prime}} \\
& =\left(1+\epsilon^{2}\right)\left\{B^{\prime}\left[-(1+\alpha)+\left(2 P_{+}+2 \alpha P_{-}\right)\right]\right\} \\
& +\epsilon^{2}\left\{B^{\prime \prime \prime}\left[\left(1+\alpha^{3}\right)-\frac{11}{4}\left(P_{+}+\alpha^{2} P_{-}\right)+\frac{3}{2}\left(\sigma_{+} P_{+}^{2}+\alpha^{3} P_{-}^{2}\right)\right]\right. \\
& \left.+E^{\prime \prime}\left[\left(1-\alpha^{2}\right)-\frac{3}{2}\left(P_{+}-\alpha^{2} P_{-}\right)\right]+3(1+\alpha) B B^{\prime}\right\}+O\left(\epsilon^{4}\right) .
\end{aligned}
$$

Since the lowest-order terms cancel, (85) can be written as follows:

$$
\begin{aligned}
& B^{\prime \prime \prime}\left[\left(1+\alpha^{3}\right)-\frac{11}{4}\left[P_{+}+\alpha^{3} P_{-}\right)+\frac{3}{2}\left(\sigma_{+} P_{+}^{2}+\alpha^{3} \sigma_{-} P_{-}^{2}\right)\right] \\
& +E^{\prime \prime}\left[\left(1-\alpha^{2}\right)-\frac{3}{2}\left(P_{+}-\alpha^{2} P_{-}\right)\right]+3(1+\alpha) B^{\prime}-(1+\alpha) B^{\prime}=0\left(\epsilon^{2}\right) .
\end{aligned}
$$

Now we eliminate $E$ from (86) by use of (84). In this way we find a differential equation for $B(x)$ of the following 
form (neglecting terms which are $O\left(\epsilon^{2}\right)$ ).

(87) $R B^{\prime \prime \prime}+3 B B^{\prime}-B^{\prime}=0$.

The coefficient $R$ is quite lengthy but can be simplified somewhat if the term containing $A^{2} / c^{2}$ in (84) is neglected. Neglecting this term is equivalent to assuming charge neutrality, instead of using Poisson's equation. With this simplification the coefficient $R$ becomes

(88)

$$
\begin{aligned}
R & =\alpha+\frac{(1-12 \alpha)}{4(1+\alpha)}\left(P_{+}-\alpha^{2} P_{-}\right) \\
& +\frac{1}{4(1+\alpha)^{2}}\left\{6(1+\alpha)\left(\sigma_{+} P_{+}^{2}+\alpha^{3} \sigma_{-} P_{-}^{2}\right)-9\left(P_{+}-\alpha^{2} P_{-}\right)^{2}\right\} .
\end{aligned}
$$

It is convenient to rewrite (88) in terms of $\beta_{+}, \beta_{-}$, which are defined as the ratios of the pressures of the ion gas and electron gas, respectively, to the magnetic pressure. That is,

$$
\beta_{+}=\frac{p_{+}}{B_{0}^{2} / 2 \mu} \quad, \quad \beta_{-}=\frac{p_{-}}{B_{0}^{2} / 2 \mu} .
$$

We have also

(90)

$$
P_{+}=\frac{(1+\alpha) \beta_{+}}{2\left(1+\epsilon^{2}\right)\left(1+\beta_{+}+\beta_{-}\right)} \quad, \quad \alpha P_{-}=\frac{(1+\alpha) \beta_{-}}{2\left(1+\epsilon^{2}\right)\left(1+\beta_{+}+\beta_{-}\right)}
$$

Inserting these expressions into (88), we obtain

$$
\begin{aligned}
R & =\alpha+\frac{(1-12 \alpha)\left(\beta_{+}-\alpha \beta_{-}\right)}{8\left(1+\beta_{+}+\beta_{-}\right)} \\
& +\frac{1}{16}\left\{6(1+\alpha)\left(\sigma_{+}+\beta_{+}^{2}+\alpha \sigma_{-} \beta_{-}^{2}\right)-9\left(\beta_{+}-\alpha \beta_{-}\right)^{2}\right\}
\end{aligned}
$$


We may assume

$$
\alpha \ll 1
$$

since the mass of an electron is much less than the mass of any positive ion. If we also assume that the ion and electron temperatures are equal, so that the pressures are equal, we have

$$
\beta_{+}=\beta_{-}=\frac{I}{2} \beta
$$

For small $\beta$, which is an interesting case, (91) reduces to

$$
R=\alpha+\frac{\beta}{16}
$$

Comparing this with the expression we get if we do not neglect $\mathrm{A}^{2} / \mathrm{C}^{2}$, we see that a necessary condition for the validity of the charge-neutral assumption is

$$
\frac{A^{2}}{c^{2}} \ll\left(\alpha+\frac{\beta}{16}\right) \text {. }
$$


6. Width of the Solitary wave

The differential equation (87) can be solved by quadratures. The solution satisfying the condition

$$
\text { for } x \rightarrow-\infty, \quad B \rightarrow 0
$$

is

$$
B(x)=\frac{1}{\cosh ^{2}\left[\frac{x}{2 R^{1 / 2}}\right]}
$$

(apart from a constant which may be added to $x$; this, however, may be removed by a shift of coordinates).

Plotted against $x$, this is a symmetric curve, rising from $B=0$ at $x=-\infty$ to its maximum $B=1$ at $x=0$, and then again falling to $B=0$ at $x=+\infty$. And $B$ takes on half its maximum value at the two points where

$$
\cosh \left(\frac{1}{2 R^{1 / 2}}\right)=2^{1 / 2}
$$

that is, at the two points

$$
x= \pm 2 R^{1 / 2} \times 0.881
$$

Now by (14), (15), (16) defining the dimensionless variables $x$, we obtain from this the following expression for the width $I$ of the solitary wave:

$$
L \approx \frac{3.52}{\sqrt{M^{2}-1}} \sqrt{1+\frac{1}{16}\left(\frac{m_{+}}{m_{-}}\right) \beta} \frac{\sqrt{m_{-} m_{+}} U_{O}}{e_{0}}
$$


which should be accurate for small (positive) values of $\left(M^{2}-1\right)$, and small $\beta$, if the condition (93) for the validity of the charge-neutral assumption obtains. Of course, under these assumptions $U_{0}$ is nearly the Alfven speed, so we have the following alternative for $\mathrm{L}$ :

$$
L \approx \frac{3.52}{\sqrt{M^{2}-1}} \sqrt{1+\frac{1}{16}\left(\frac{m_{+}}{m_{-}}\right) \beta} \quad \frac{1}{e} \sqrt{\frac{m_{-}}{\mu n_{0}}}
$$

The analogous formula for the cold plasma -- which can also be obtained directly (our earlier work) -- is found by setting $\beta=0$.

Thus we see that the effect of plasma temperature on the Adlarn-Allen solitary wave is to multiply the width of the wave by the factor

$$
\sqrt{1+\frac{1}{16}\left(\frac{m_{+}}{m_{-}}\right) \beta},
$$

for small $\beta$. 

1. J. H. Adlam and J. E. Allen, "The structure of strong collision-free hydromagnetic waves", Phil. Mag. 3, 448-456 (1958).

2. A. Banos and A. R. Vernon, "Large amplitude waves in a collision-free plasma I. Single pulses with isotropic pressure", Nuovo Cimento 15, 269-288 (1960).

3. G. F. Chew, M. I. Goldberger and F. E. Low, "The Boltzmann equation and the one-fluid hydromagnetic equation in the absence of particle collisions", Proc. Royal Society, Series A, 236, $112-118$ (1956).

4. L. Davis, R. Llist and A. Schlltter, "The structure of hydromagnetic shock waves $I$. Non-linear hydromagnetic waves in a cold plasma", Zeitschrift fur Naturforschung 13a, 916-936 (1958).

5. C. S. Gardner, H. GoertzeI, H. Grad, C. S. Morawetz, M. H. Rose and $\mathrm{H}$. Rubin, "Hydromagnetic shock waves in hichtemperature plasmas", Proceedings of the Second United Nations International Conf. On the Peaceful Uses of Atomic Energy, Vol. 31, 230-237 (1958).

6. C. S. Gardner and G. K. Morikawa, "Similarity in the asymptotic behavior of collision-free hydromagnetic waves and water waves", TID-6184, Institute of Mathematical Sciences, New York Univ. (May 1960).

7. H. Grad, "On the kinetic theory of rarified gases", Comm. Pure Appl. Math. 2, 331 (1949).

8. H. Grad, "General adiabatic theory", Controlled Thermonuclear Conf., Gatlinburg, Tenn., TID-7520, washington, D. C., p. 402 $(1956)$.

9. J. B. Keller, "The solitary wave and periodic waves in shallow water", Comm. Pure Appl. Math. 1, 323-339 (1948).

10. D. J. Korteweg and G. de Vries, "On the change of form of long waves advancing in a rectangular canal, and on a new type of long stationary waves", Phil. Mag., Series 5, 39, 422 (1895).

11. C. S. Morawetz, "Magneto-hydradynamic shock structure without collisions," NYO-2885, Institute of Mathematical Sciences, New York Univ. (March 1960).

12. G. K. Morikawa, "Non-linear diffusion of flood waves in rivers", Comm. Pure Appl. Math. 10, 291-303 (1957). 
13. G. K. Morikawa, "Geostrophic Vortex Motion", J. of Meteorology 17, 148-158 (1960).

14. M. H. Rose, "On plasma-magnetic shocks", NYO-2883, Institute of Mathematical Sciences, New York Univ. (March 1960).

15. A. R. Vernon, "Large amplitude waves in a collision-free plasma", Report No. 3, Dept. of Physics, Univ. of Calif. at Los Angeles (1960). 


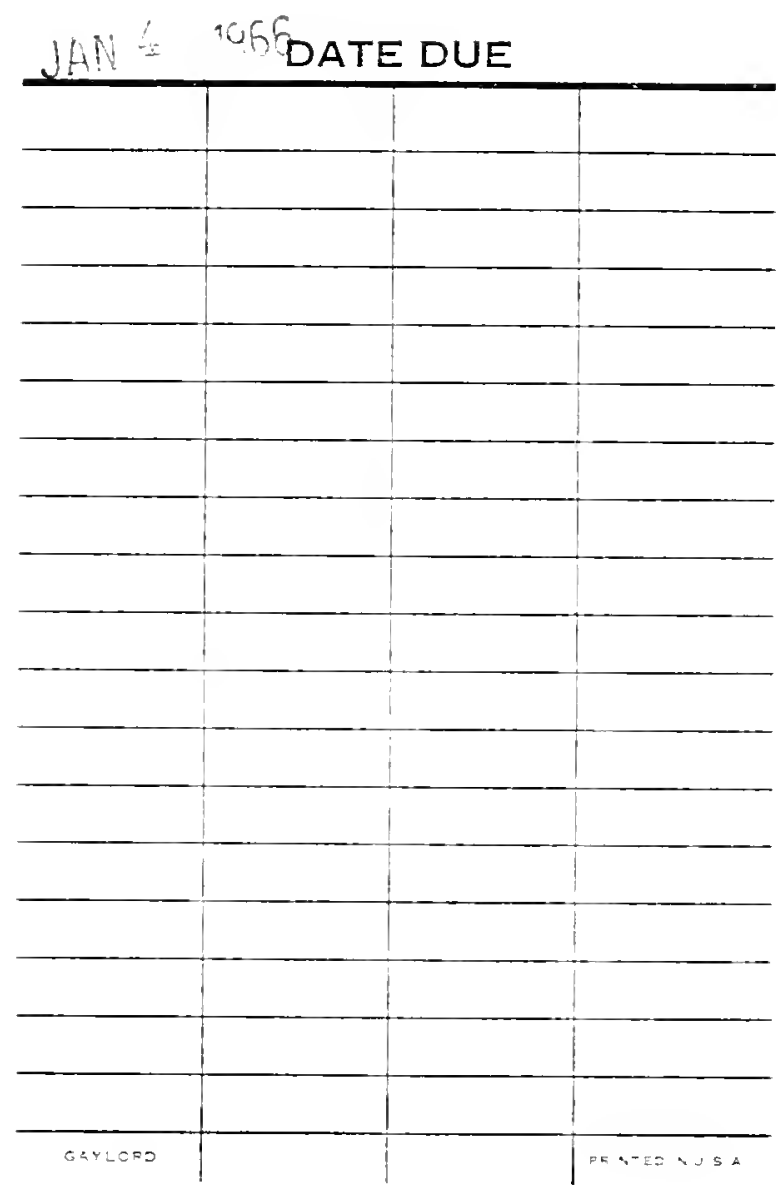




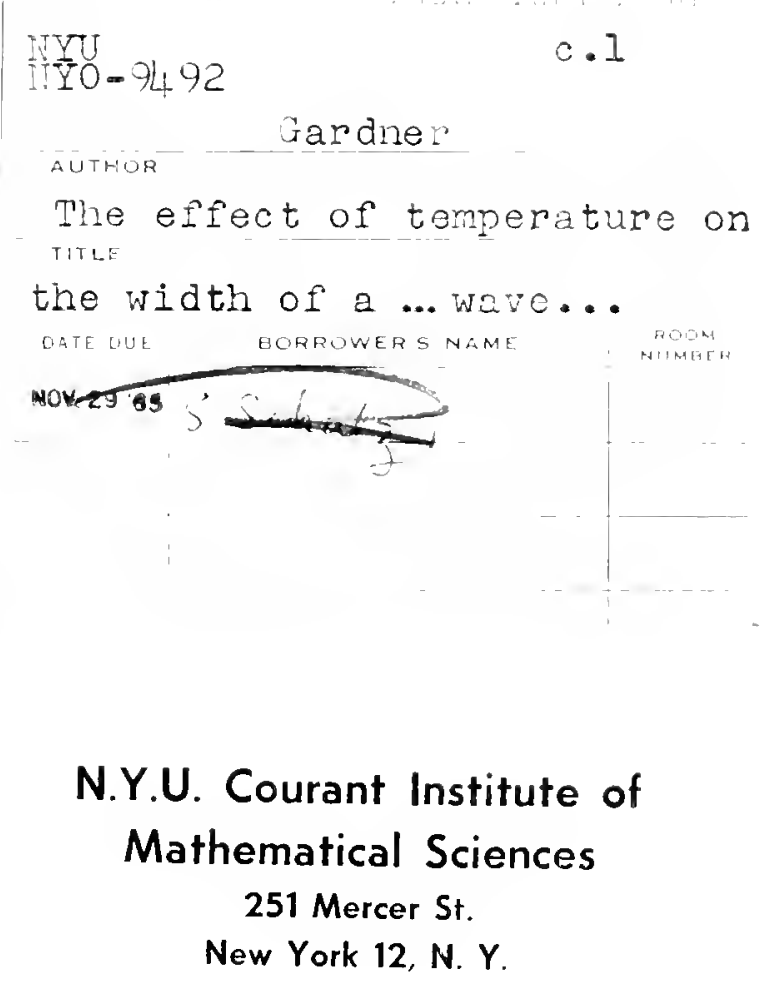


